\title{
Experimental model in rats of penumbra area using implemented baloon in caudate nucleus volume dependent and time dependent
}

\begin{abstract}
The study aims to determine whether early removal of an experimental intracerebral mass alters neurological function. In four experimental series, $0.6 \mathrm{~mL}$ and $0.8 \mathrm{~mL}$ balloons were implanted by stereotaxis and inflated into the right caudate nucleus of rats. After lhour of insufflation, the brains were removed and studied by histopathological analysis. Immunohistochemical was included with protein S-100, marker of neuronal destruction. Four groups were formed (1A, 1B, 2A, 2B) and the variables: time and balloon volume was analyzed. In each series of the time variable, half of the animals had a $0.8 \mathrm{~mL}$ balloon inflated for three minutes (Group 1A), and the other half had a $0.8 \mathrm{~mL}$ balloon inflated for ten minutes (Group 2A). In the variable balloon volume series, half of the animals had a $0.6 \mathrm{~mL}$ balloon inflated for 6 minutes (Group 1B), while the other half had a $0.8 \mathrm{~mL}$ balloon inflated for 6minutes (Group 2B). In the qualitative and quantitative analysis of the parameters, the shortest group (group 1A), either there was no lesion, or there was partial loss of nervous tissue, while the longest group (group 2A) presented edema and cerebral parenchymal necrosis, reaching $35 \%$ of nuclear pyknosis. In the volume dependent groups (groups $1 \mathrm{~B}$ and $2 \mathrm{~B}$ ), the findings were similar, both with about $30 \%$ of pyknotic nuclei. Thus, time was the major determinant of injury, reiterating the prognostic importance of early removal of a spontaneous intracerebral mass.
\end{abstract}

Keywords: brainischemia, Caudate nucleus, Immunohistochemical, intracerebral mass, S100 proteins
Volume 9 Issue 5 - 2019

\author{
João Pedro Franco Leal de Oliveira,' Tulio \\ Melo Oliveira,' Vitor Pelogi Arienzo,' \\ Henrique Alvares Pessoa, 'Valquiria Neves \\ Perin,' Willy França, ${ }^{2}$ Maria Lourdes Peris \\ Barbo, ${ }^{3}$ Luciana Canabarro, ${ }^{2}$ Paulo Henrique \\ Pires de Aguiar ${ }^{4}$ Isabella Rita Fadel Martinho ${ }^{4}$ \\ 'Student of medicine graduation at Pontifical Catholic University \\ of Sao Paulo, Brazil \\ 2Discipline of Surgical Technique of Pontifical Catholic University \\ of Sao Paulo, Brazil \\ ${ }^{3}$ Discipline of Pathology of Pontifical Catholic University of Sao \\ Paulo, Brazil \\ ${ }^{4}$ Discipline of Neurology of Pontifical Catholic University of Sao \\ Paulo, Brazil
}

Correspondence: Paulo Henrique Pires de Aguiar, Professor of the medical course of the, Pontifical Catholic University of São Paulo, Brazil, Email phpaneurocir@gmail.com

Received: September 08, 2019 | Published: September 18, 2019

\section{Introduction}

Stroke is the second leading cause of death in the world, second only to cardiovascular disease. ${ }^{1}$ In Brazil, however, strokes symbolize the leading cause of death, then, by acute myocardial infarction. The incidence of strokes increases progressively from the eighth decade of life onwards, similarly in both sexes. ${ }^{2}$ According to the World Health Organization (WHO), by 2030 stroke remains the second leading cause of death representing $12.2 \%$ of predicted deaths for the year. ${ }^{3}$

Stroke reflects high levels of morbidity and mortality, and when the patient does not die, it is very likely to have temporary or permanent functional disabilities. ${ }^{4}$ There are two classic forms of stroke: ischemic (vessel obstruction, hindering the supply of oxygen and substrates to brain tissue - the result of atherosclerotic or embolic processes) and hemorrhagic (extravasation of blood into or around central nervous system - intraparenchymal and subarachnoid structures, respectively). ${ }^{5}$

After spontaneous intracerebral hemorrhage, several patients die or become assiduously impaired. However, although this dire prognosis sometimes results from a late deterioration, it is still controversial the effectiveness of lesion early removal. ${ }^{6-11}$

Thus, in 1990, Nehls et al. ${ }^{12}$ presented in the Congress of Neurological Surgeons the journal: "Experimental Intracerebral Hemorrhage: Early Removal of a Spontaneous Mass Lesion Improves Late Outcome", ${ }^{12}$ in which is discussed the validity of the early removal of the lesion caused by a spontaneous mass. This study is based on this experimental work and seeks to be based on current literature over penumbra area. ${ }^{13-18}$
Finally, having interpreted and compared the findings and histopathological alterations, the paper elucidates that in the groups of rats subjected to transient balloon inflation, the differences in blood flow from each cerebral hemisphere were surprisingly small. However, for the groups of rats subjected to the permanent balloon, blood flow rates were significantly worse in the ipsilateral balloon hemisphere: the main and mean levels of caudate nucleus blood flow; the main blood flow of the cerebral cortex; and blood flow in the area of cortical ischemia. Specific gravity was reduced in the areas surrounding the balloon insertion site after both temporary and permanent inflation and there was evidence of ischemic cell injury in all animals studied.

Therefore, based on this journal, this research proposed to analyze if the early removal of an experimental intracerebral mass brings benefits to the patient. However, this study differs through immunohistochemical analysis with the use of protein S-100, which is expressed by astroglia in the brain and accumulates around ischemic lesions. ${ }^{19}$ In addition, through stereotaxis, it was studied from the perspective of not just one variable, but two: the time and volume of the inflated balloon.

It is worth highlighting that stereotaxis is a technique employed since the twentieth century, which allows the introduction of brain implants precisely through a coordinate system. Currently, it is useful in animal experiments, especially rodents. ${ }^{20}$

\section{Objectives}

The objective of the present study was to analyze whether early removal of an experimental intracerebral mass alters cerebral blood 
flow, brain water content, or neurological function after one hour and to analyze histological changes in the affected region.

\section{Methods}

The present project was submitted for evaluation of the Ethics Committee on Animal Use (CEUA) of the FCMS-PUC/SP and subsequently approved by the same- $n^{\circ} 2017 / 83$.

Twenty adult (Wistar) rats weighing approximately 600 and 800 grams, aged from 6 months to 1year, were placed in cages kept in a controlled temperature environment $\left(25 \pm 2{ }^{\circ} \mathrm{C}\right)$ and exposed to light for a daily period of 12 hours (7:00-19:00h). The animals had access ad libitum to the commercial ration. The animals used in this study were from the animal health Sciences Faculty of PUC-SP (FCMS, PUC/SP). On the day of the experiment, the animals were submitted to sedation with ketamine base- $50 \mathrm{mg} / \mathrm{ml}$ (Ketalar $\left.{ }^{\circledR}\right)$ at a dose of $35-40 \mathrm{mg} / \mathrm{kg}$, Intramuscular, associated with xylazine $10 \mathrm{mg} /$ $\mathrm{ml}$ (Coopazine $\left.{ }^{\circledR}\right)$. At a dose of $5-6 \mathrm{mg} / \mathrm{kg}$, also intramuscularly applied to the lateral musculature of the thigh with insulin syringe and $20 \mathrm{G}$ needle. These associated drugs cause a sedative effect that lasts around lhours, long enough to perform the surgery.

Then, they were placed in a stereotactic apparatus. The skull was exposed and a trepanation hole was made with refrigerated saline dental drill at a point located on the right caudate nucleus, using stereotactic coordinates, which were: on the anteroposterior axis, 1 , $3 \mathrm{~mm}$ caudal relative to the line Interaural; on the side-lateral axis, $1,6 \mathrm{~mm}$ towards the right side; in the dorsoventral axis, $3,46 \mathrm{~mm}$ superiorly to the Interaural line, previously established by the consultation with the Stereotactic Atlas.

A 15-Fogart F-4 Micro balloon was mounted at the end of a 23-gauge needle, connected to a Micro syringe (Hamilton Bonaduz AG, Bonaduz, Switzerland). The balloons were inserted in the center of the caudate nucleus by Stereotactic apparatus (Narishige, Japan) following the pattern of Stereotactic Atlas for Rats (Paxinos and Watson, 1986). Then, the balloons were inflated according to the experimental groups.

a. The materials used in the procedure were all sterilized in an autoclave system.

b. The animals were divided into 4 groups, described below:

c. Groups (1A, 2A, 1B, 2B).

Group 1A: Consists of the use of 5 animals, in which the time variable was analyzed by inflation of a $0.8 \mathrm{~mL}$ balloon for 3 minutes.

Group 2A: Consists of the use of 5 animals, in which the time variable was analyzed by inflation of a $0.8 \mathrm{~mL}$ balloon for 10 minutes.

Group 1B: Consists of the use of 5 animals, in which the variable balloon volume was analyzed by inflation of a $0.8 \mathrm{~mL}$ balloon for 6 minutes.

Group 2B: Consists of the use of 5 animals, in which the variable balloon volume was analyzed by inflation of a balloon of $0,6 \mathrm{~mL}$ balloon for 6 minutes.

\section{Qualitative neuropathological studies}

After being sacrificed, the rats had their brains removed and stored in a solution of acetic formaldehyde and methoic acid. Subsequently, the brains were sent for histological analysis according to the techniques used for light microscopy, using paraffin as a means of inclusion and staining by Hematoxylin, eosin and a combination of fast blue Luxol with Cresyl Violet. The Immunohistochemical method was used in paraffin sections with buffer solution and an anti-protein antibody S-100 (marker of neuronal death), by means of avidin biotin or immunoperoxidase, analyzed with Chromogen diaminobenzidine under magnification Microscopic number of cells stained with S-100.

The immune-histopathological analysis was performed by the Laboratory of Pathology and Cytology of Sorocaba and the laboratory of Molecular Biology of the Faculty of ABC.

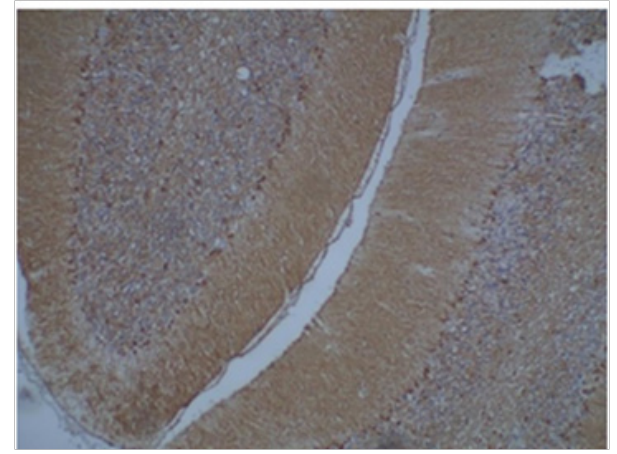

Figure I Rat belonging to group IA - hemisphere ipsilateral to balloon inflation with S-I00 staining - conserved brain tissue. Sorocaba/SP/Brazil, $2017 / 2018$.

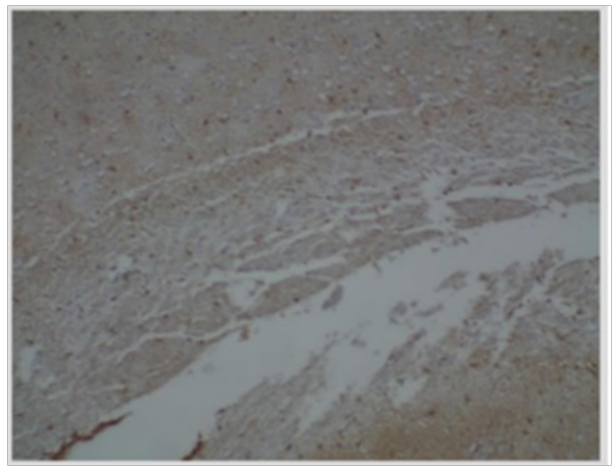

Figure 2 Rat belonging to group IA- hemisphere ipsilateral to balloon inflation with S-100 staining, discreet edema with necrosis. Sorocaba/SP/Brazil, $2017 / 2018$

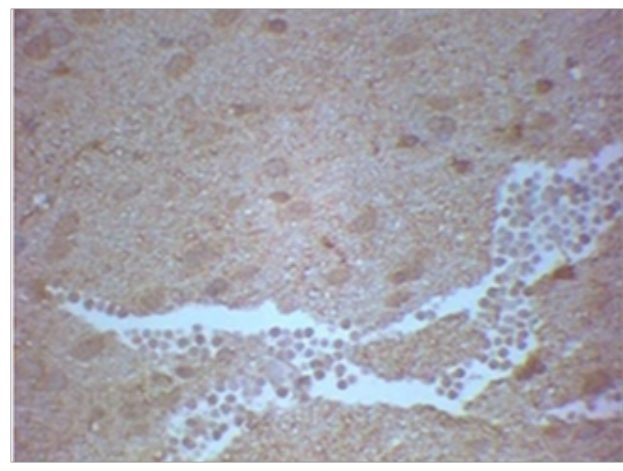

Figure 3 Rat belonging to group 2A - hemisphere ipsilateral to balloon inflation with S-I00 staining - extensive edema with brain tissue necrosis and nuclear pyknosis. Sorocaba/SP/Brazil, 20I7/20I8 


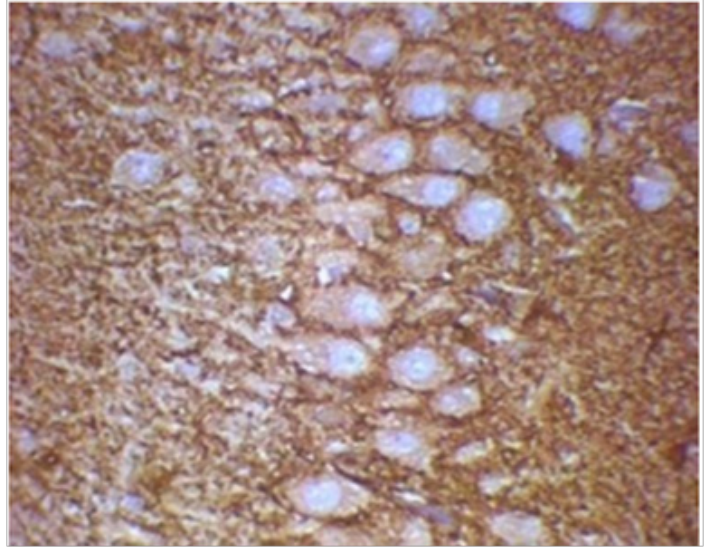

Figure 4 Rat belonging to group 2A - hemisphere ipsilateral to balloon inflation with S-100 staining - presence of intense edema, loss of cell boundaries, characterizing necrosis with Purkinje cell destruction and nuclear pyknosis. Sorocaba/SP/Brazil, 20I7/20I8

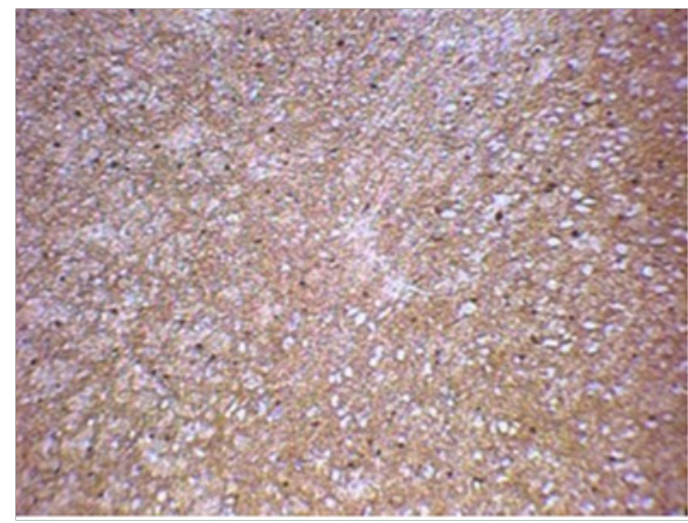

Figure 5 Rat belonging to group IB - hemisphere ipsilateral to balloon inflation with S-100 staining - extensive edema and necrosis of nervous tissue. Sorocaba/SP/Brazil, 2017/2018

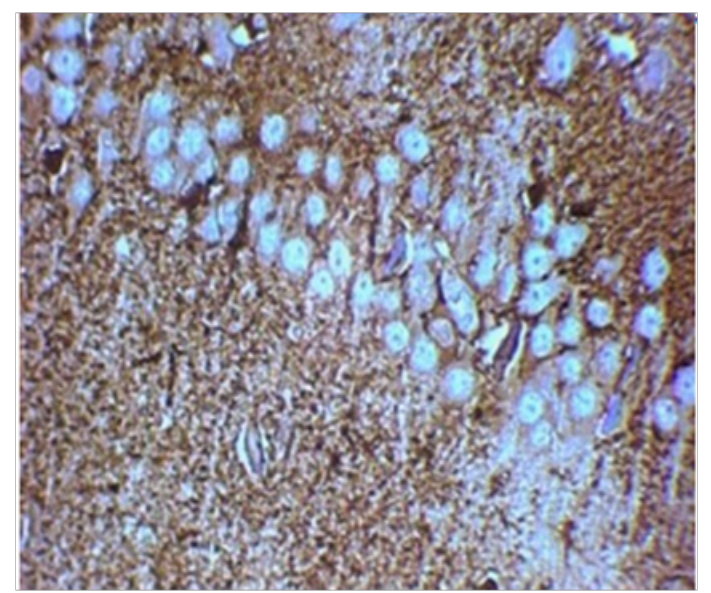

Figure 6 Rat belonging to group IB - hemisphere ipsilateral to balloon inflation with S-100 staining - Purkinje cell necrosis with nuclear pyknosis. Sorocaba/SP/Brazil, 2017/2018

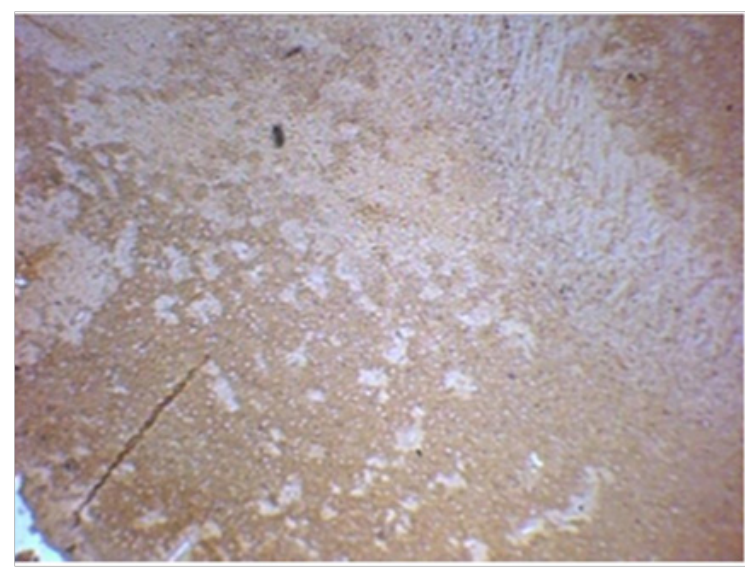

Figure 7 Rat belonging to group 2B - hemisphere ipsilateral to balloon inflation with S-100 staining - strong edema and partial necrosis of nervous tissue. Sorocaba/SP/Brazil, 2017/2018

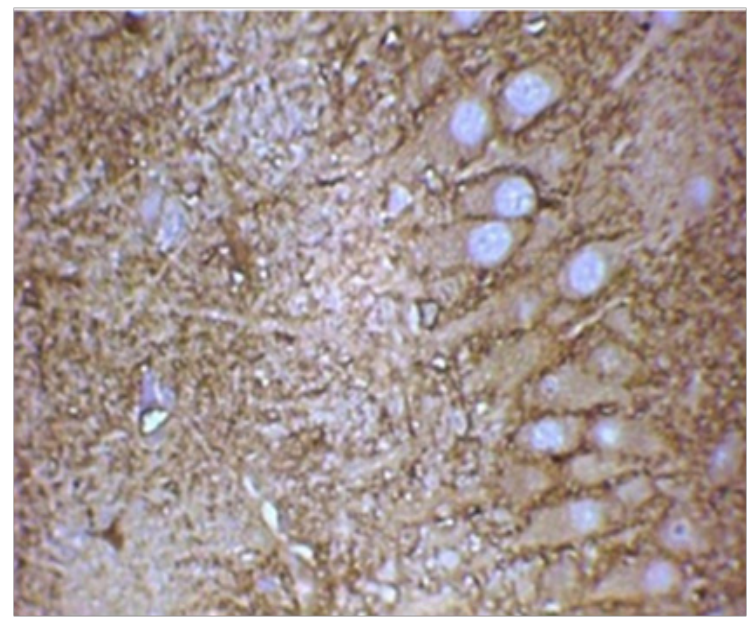

Figure 8 Rat belonging to group 2B - hemisphere ipsilateral to balloon inflation with S-I00 staining - edema and necrosis of nervous tissue, with Purkinje cell apoptosis and with pyknosis of nuclei. Sorocaba/SP/Brazil, 20I7/20I8

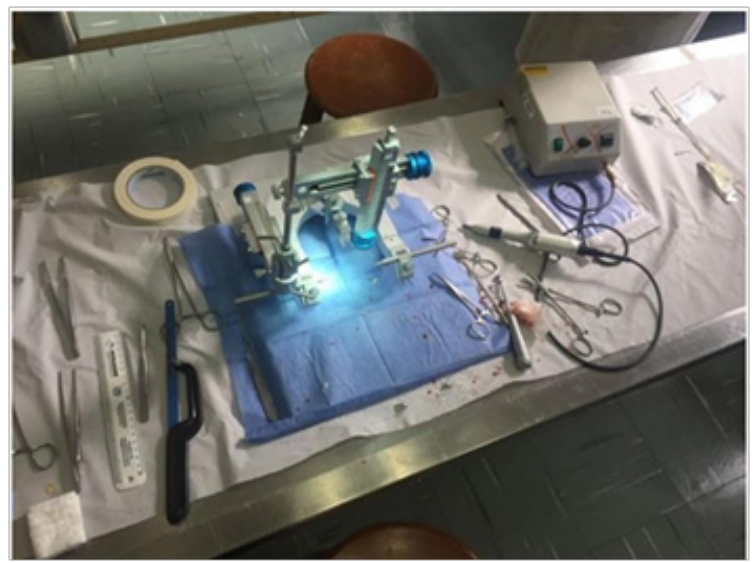

Figure 9 Stereotactic apparatus and other materials for neurosurgery. Sorocaba/SP/Brazil, 2017/2018 


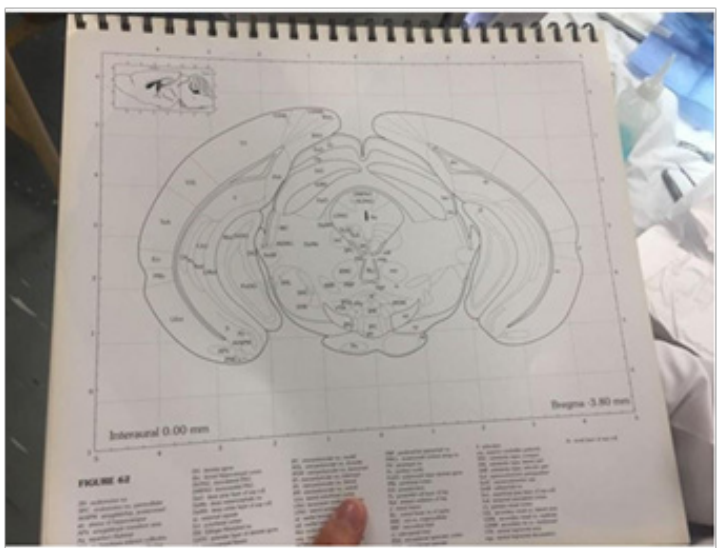

Figure 10 Stereotactic Atlas for Rats. Sorocaba/SP/Brazil, 2017/2018

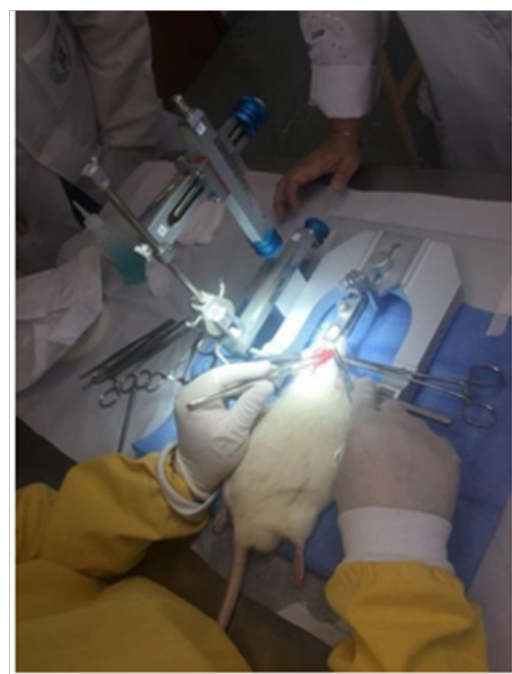

Figure II Start of surgery by scalping the rat - access to the skull. Sorocaba/ SP/Brazil, 2017/2018

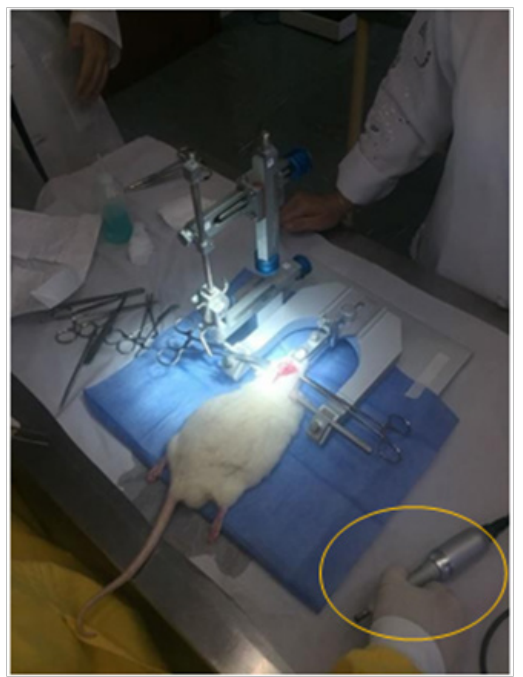

Figure 12 Drill for cranial perforation at the location indicated by the stereotactic atlas. Sorocaba/SP/Brazil, 2017/2018

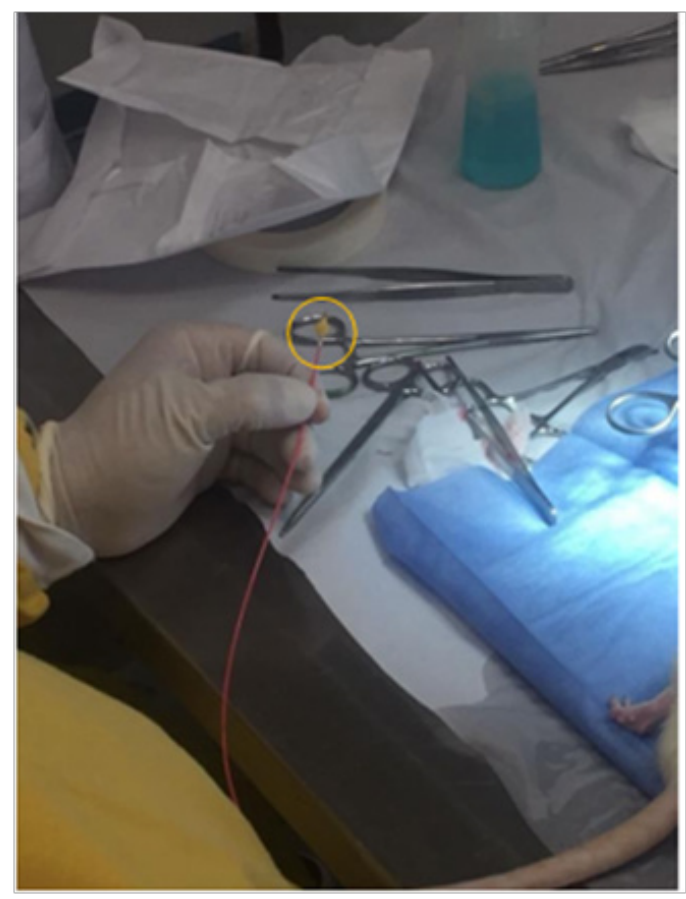

Figure I 3 Microballoon I5 Fogart F-4. Sorocaba/SP/Brazil, 2017/2018

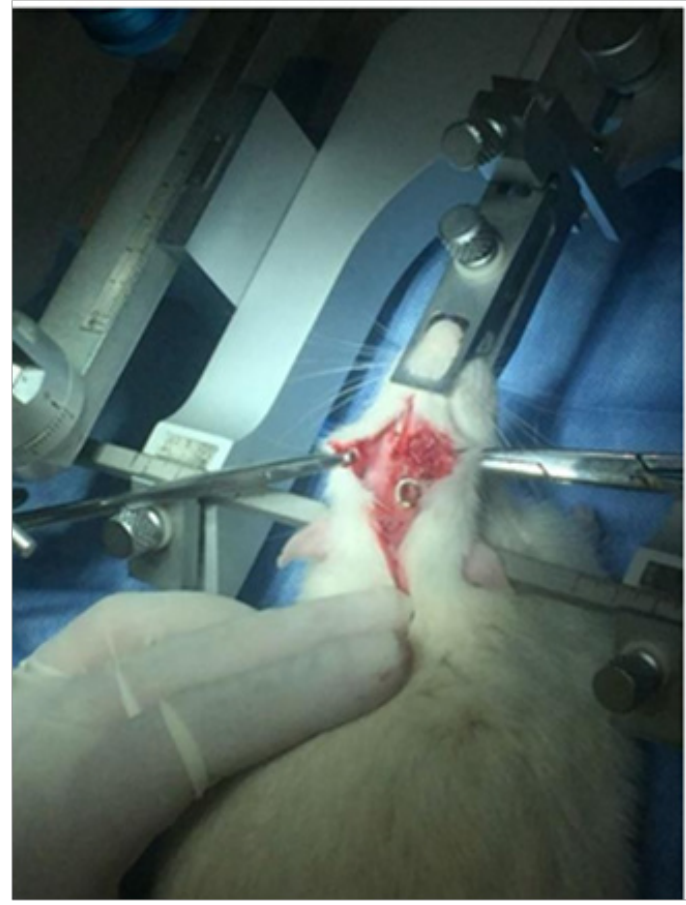

Figure I4 Rat positioned in stereotactic apparatus after inflation of the respective balloons. Sorocaba/SP/Brazil, 2017/2018

\section{Results}

During the project, a total of twenty Wistar rats were submitted to the stereotactic procedure and their brains were analyzed and compared by histopathological findings and immunohistochemical analysis of S-100 protein. Thus, each study group had a $\mathrm{N}$ of 5 rats. 
The following will be presented, in full, the data obtained in the histopathologic and immunohistochemical analysis of the hemisphere ipsilateral to balloon inflation:

\section{Analysis of the hemisphere ipsilateral to balloon inflation}

Group 1A: Consists of the use of 5 animals, in which the time variable was analyzed by inflation of a $0.8 \mathrm{~mL}$ balloon for 3 minutes (Table 1 ).
Group 2A: Consists of the use of 5 animals, in which the time variable was analyzed by inflation of a $0.8 \mathrm{~mL}$ balloon for 10 minutes (Table 2 ).

Group 1B: Consists of the use of 5 animals, in which the variable balloon volume was analyzed by inflation of a $0.8 \mathrm{~mL}$ balloon for 6minutes (Table 3).

Group 2B: Consists of the use of 5 animals, in which the variable balloon volume was analyzed by inflation of a balloon of $0,6 \mathrm{~mL}$ balloon for 6 minutes (Tables 4 \& 5).

Table I Analysis of the ipsilateral hemisphere to balloon inflation in group IA, Sorocaba/SP/Brazil, 20I7/20I8

\begin{tabular}{llll}
\hline & Edema formation & Presence of necrosis & Pyknotic nuclei \\
\hline Rat I & Absence of edema, preserved brain area & Absence of necrosis & Absence of pyknotic nuclei \\
Rat 2 & Discrete edema & $\begin{array}{l}\text { Partial necrosis of } \\
\text { Brain tissue }\end{array}$ & $\begin{array}{l}\text { Presence of 25\% of pyknotic } \\
\text { nuclei }\end{array}$ \\
Rat 3 & Absence of edema, preserved brain area & Absence of necrosis & Absence of pyknotic nuclei \\
Rat 4 & Discrete edema & Partial necrosis of & Presence of 25\% of pyknotic \\
& & Brain tissue & nuclei \\
Rat 5 & Discrete edema & Partial necrosis of & Presence of 25\% of pyknotic \\
& & Brain tissue & nuclei
\end{tabular}

Table 2 Analysis of the ipsilateral hemisphere to balloon inflation in group 2A, Sorocaba/SP/Brazil, 2017/20I8

\begin{tabular}{llll}
\hline \multicolumn{1}{c}{ Edema formation } & Presence of necrosis & Pyknotic nuclei \\
\hline Rat I Extensive brain parenchyma edema & Accentuated brain tissue necrosis & Presence of $35 \%$ of pyknotic nuclei \\
Rat 2 Extensive brain parenchyma edema & Accentuated brain tissue necrosis & Presence of $35 \%$ of pyknotic nuclei \\
Rat 3 Cerebral tissue strongly edematous & Intense necrosis with Purkinje cells loss & Presence of $35 \%$ of pyknotic nuclei \\
Rat 4 Extensive brain parenchyma edema & Accentuated brain tissue necrosis & Presence of $35 \%$ of pyknotic nuclei \\
Rat 5 Cerebral tissue strongly edematous & Intense necrosis with Purkinje cells loss & Presence of $35 \%$ of pyknotic nuclei \\
\hline
\end{tabular}

Table 3 Analysis of the ipsilateral hemisphere to balloon inflation in group 1B, Sorocaba/SP/Brazil, 2017/2018

\begin{tabular}{llll}
\hline & Edema formation & Presence of necrosis & Pyknotic nuclei \\
\hline Rat I & Extensive brain parenchyma edema & Intense necrosis with Purkinje cells loss & Presence of $30 \%$ of pyknotic nuclei \\
Rat 2 & Extensive brain parenchyma edema & Accentuated brain tissue necrosis & Presence of $30 \%$ of pyknotic nuclei \\
Rat 3 & Extensive brain parenchyma edema & Intense necrosis with Purkinje cells loss & Presence of $30 \%$ of pyknotic nuclei \\
Rat 4 & Cerebral tissue strongly edematous & Accentuated brain tissue necrosis & Presence of $30 \%$ of pyknotic nuclei \\
Rat 5 & Extensive brain parenchyma edema & Intense necrosis with Purkinje cells loss & Presence of $30 \%$ of pyknotic nuclei \\
\hline
\end{tabular}

Table 4 Analysis of the ipsilateral hemisphere to balloon inflation in group 2B, Sorocaba/SP/Brazil, 20I7/20I8

\begin{tabular}{llll}
\hline & Edema formation & Presence of necrosis & Pyknotic nuclei \\
\hline Rat I & Cerebral tissue strongly edematous & Accentuated brain tissue necrosis & Presence of $30 \%$ of pyknotic nuclei \\
Rat 2 & Extensive brain parenchyma edema & Intense necrosis with Purkinje cells loss & Presence of $30 \%$ of pyknotic nuclei \\
Rat 3 & Extensive brain parenchyma edema & Intense necrosis with Purkinje cells loss & Presence of $30 \%$ of pyknotic nuclei \\
Rat 4 & Extensive brain parenchyma edema & Accentuated brain tissue necrosis & Presence of $30 \%$ of pyknotic nuclei \\
Rat 5 & Cerebral tissue strongly edematous & Accentuated brain tissue necrosis & Presence of $30 \%$ of pyknotic nuclei
\end{tabular}

Table 5 Average Pyknotic Nuclei. Sorocaba/SP/Brazil, 2017/2018

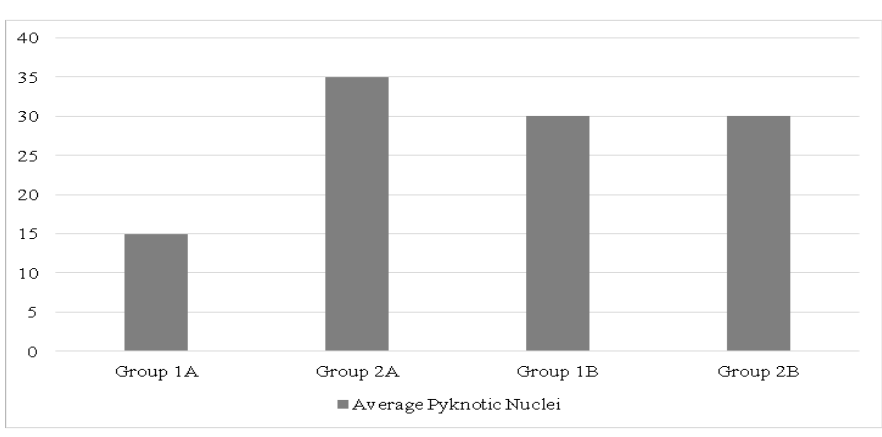




\section{Discussion}

The study aimed to determine whether the early removal of an experimental intracerebral mass alters cerebral blood flow, cerebral water content, neuropathological findings or neurological function after 1 hour. Thus, two variables were used, the balloon inflation time and the balloon inflation volume.

After histopathological investigation in addition to the immunohistochemical of S-100 protein, a qualitative analysis and quantitative analysis of the blades was performed, concluding that the variable time is the one that exerts the greatest impact on the extent and repercussion of the brain injury.

In the 5 brains of group 1A, that is, those in which the balloon was inflated with a volume of $0.8 \mathrm{~mL}$ for only 3 minutes, was where the lowest rate of brain injury was observed. Two of the five rats had their respective nervous tissues fully preserved and the remaining three presented significantly milder edema and necrosis than groups $2 \mathrm{~A}, 1 \mathrm{~B}$ and $2 \mathrm{~B}$ in the hemisphere ipsilateral to balloon inflation. Thus, they obtained a nuclear pyknosis average of only $15 \%$.

The group $2 \mathrm{~A}$, that is, the one in which the balloon was inflated with a volume of $0.8 \mathrm{~mL}$ for 10 minutes, was, precisely, which showed a higher degree of loss of brain parenchyma. The 5 brains of this group suffered intense necrosis and edema in the hemisphere ipsilateral to the balloon implantation and about $35 \%$ of their nuclei became pyknotic, thus representing the highest mean of nuclear pyknosis of the 4 study groups. Regarding the volume, there were no notable differences between one group and the other. The results were extremely similar. In group $1 \mathrm{~B}$, the one in which the balloon was inflated with a volume of $0.8 \mathrm{~mL}$ for 6 minutes, the 5 brains revealed important necrosis and edema in their hemispheres ipsilateral to the balloons and obtained about $30 \%$ of nuclear pyknosis.

In group $2 \mathrm{~B}$, the one in which the balloon was inflated with a volume of only $0.6 \mathrm{~mL}$ for 6 minutes, four brains suffered extensive necrosis and edema, and only 1 brain had partial necrosis of the nervous tissue. The mean nuclear pyknosis was also about $30 \%$.

Thus, it was evidenced that the balloon inflation time was the greatest determinant in the size and vastness of the lesion, while the volume little distinction caused. At the shorter time group, that is, 3 minutes of inflation, it was where less damage to the nervous tissue was visualized and the at the longest time group, which corresponds to 10 minutes of inflation, was, explicitly, the most injury to the cerebral parenchyma.

Therefore, it reinforces the result shown in the "Experimental intracerebral mass: time related effects on local cerebral blood flow", ${ }^{14}$ the greater the inflation time of the balloon, the greater the area of Penumbra formed. In other words, more severe is the ischemic lesion of the cerebral parenchyma.

However, when confronting the "Early hemodynamic changes in experimental intracerebral hemorrhage", ${ }^{16}$ it is possible to identify that the volume of a cerebral hemorrhagic lesion implies an impact greater than the volume of a mass lesion. In this study, the variation in the volume of the balloon had little to do with regard to cerebral pyknosis and loss of neuronal density and in the "Early hemodynamic changes in experimental intracerebral hemorrhage" 16 it was attested that the degree of ischemia by bleeding brain was volume dependent.

Finally, the findings of this research reiterate the discovers of the paper "Experimental Intracerebral Hemorrhage: Early Removal of a
Spontaneous Mass Lesion Improves Late Outcome", ${ }^{12}$ stating that the earlier is the removal of an intracerebral mass, the lower the area of penumbra.

\section{Conclusion}

Thus, it is reiterated how fundamental it is for the prognosis of the patient to early withdrawal of a spontaneous intracerebral mass. It would be extremely interesting to continue and deepen in this project, in order not only to analyze the histopathological findings, but also with a real-time cerebral flow meter. In this way, it could be precisely measured the changes in the cerebral circulation as a function of balloon inflation.

\section{Acknowledgments}

None.

\section{Conflicts of interest}

The authors declare that there are no conflict of interest.

\section{Funding}

None.

\section{References}

1. World Health Organization (WHO). The top 10 causes of death. WHO: Geneva; 2014.

2. Ministry of Health. Health Information TABNET - Vital Statistics. 2017.

3. World Health Organization (WHO). Health statistics and information systems - Projections of mortality and causes of death, 2015 and 2030. WHO: Geneva; 2013.

4. Damata SR, Formiga LM, Araújo AK, et al. Epidemiological profile of elderly people with stroke. $R$ Interd. 2016;9(1):107-117.

5. Chaves ML. Stroke: conceptualization and risk factors. Rev Bras Hyperertens. 2000;7(4):372-382.

6. Arana-Iniguez R, Wilson E, Bastarrica E, et al. Cerebral hematomas. Surg Neurol. 1976;6(1):45-52.

7. Duff TS, Ayeni S, Levin A. Non-surgical management of spontaneous intracerebral hemorrhage. Neurosurgery. 1981;9:387-393.

8. Kaneko M, Tanaka K, Shimada T, et al. Longterm evaluation of ultraearly operation for hypertensive intracerebral hemorrhage in 100 cases. J Neurosurg. 1983;58(6):838-842.

9. Kanno T, Sano H, Shinomiya, et al. Role of surgery in hypertensive intracerebral hematoma: A comparative study of 305 nonsurgical and 154 surgical cases. J Neurosurg. 1984;61(6):1091-1099.

10. Mitsukami M, Tazawa T. Theoretical background for surgical treatment in hypertensive intracerebral hemorrage. In: Mizukami M, Kanaya H, editors. Hypertensive intracerebral hemorrhage. New York: Raven Press; 1983. p. 239-247.

11. Waga S, Yakamamoto Y. Hypertensive putaminal hemorrhage: treatment and results. Is surgical treatment superior to conservative one? Stroke. 1983;14(4):480-485.

12. Nehls DG, Mendelow AD, Graham DI, et al. Experimental intracerebral hemorrage: early removal of spontaneuos mass lesion improves late outcomes. Neurosurgery. 1990;27(5):674-682.

13. Bullock R, Mendelow AD, Teasdale GM, et al. Intracranial haemorrhage induced at arterial pressure in the rat: Part 1. Description of technique, ICP changes and neuropathological findings. Neurol Res. 1984;6(4):184188. 
14. Kingman TA, Mendelow AD, Graham DI. Teasdale GM. Experimental intracerebral mass: time related effects on local cerebral blood flow. $J$ Neurosurg. 1987;67:732-738.

15. Mendelow AD, Bullock R, Teasdale GM, Graham DI, McCulloch $\mathrm{J}$. Intracranial haemorrage induced at arterial pressure in the rat: Part 2. Short term changes in local cerebral blood flow measured by autoradiography. Neurol Res. 1984;6(4):189-193.

16. Naths FP, Jenkins A, Mendelow AD, et al. Early hemodynamic changes in experimental intracerebral hemorrhage. J Neurosurg. 1986;65(5):697703.

17. Nehls DG, Mendelow AD, Graham DI, et al. Experimental intracerebral hemorrhage: the progression of hemodynamic changes following production of a spontaneous mass lesion. Neurosurgery. 1998;23(4):439_ 444.

18. Sinar EJ, Mendelow AD, Graham DI, et al. Experimental intracerebral hemorrhage: effects of a temporary mass lesion. $J$ Neurosurg. 1987;66(4):568-5676.

19. Ohtani R, Tomimoto H, Wakita H, et al. Expression of S100 protein and protective effect of arundic acid on the rat brain in chronic cerebral hypoperfusion. Brain Res. 2007.1135(1):195-200.

20. De Vloo P, Nuttin B. Stereotaxy in rat models: Current state of the art, proposals to improve targeting accuracy and reporting guideline. Behavioural Brain Research. 2017.364:457-463. 